\title{
Liposomal binding of imipramine in human red cell/albumin solution with simulated plasmapharesis
}

\author{
Grant Cave ${ }^{1 *}$, Mohan Raghavan ${ }^{1}$, Julie Burrows ${ }^{2}$, Martyn Harvey ${ }^{3}$ and Anuj Chauhan ${ }^{4}$ \\ *Correspondence: grantcave@gmail.com \\ 'Tamworth Rural Referral Hospital, Tamworth, NSW, Australia. \\ ${ }^{2}$ University of Newcastle Department of Rural Health Tamworth, NSW, Australia. \\ ${ }^{3}$ Waikato Hospital Hamilton, New Zealand. \\ ${ }^{4}$ University of Florida, Gainsville, Florida.
}

\begin{abstract}
There has been much recent interest in the use of intravenous lipid based products inthe treatment of tricyclic ntidepressant toxicity. High affinity liposomal carriers have been designed which may be more effective binders of tricyclic antidepressant than intravenous lipid emulsion in the setting of intoxication. The uniformity and size of the liposomes opens the theoretical potential for extracorporeal removal of drug once bound. Imipramine was added to a suspension of human red blood cells and albumin. Liposomes of known high affinity for tricyclics were then added to this suspension. It was hypothesized that the imipramine would be bound to liposomes, which could then be filtered off in a staged process, first using a commercially available plasmapharesis filter and then through a 100nm filter. tatistically significant reductions in imipramine concentration were seen in the final components post filtration, both red cell rich (median, 24\% original level, IQR 9-43\%) and "pharesate", liposomes filtered off (median 23\%, IQR 9-37\%). We have demonstrated significant experimental binding of imipramine to liposomes ina human albumin/red blood cell uspension, and have removed liposomes from thissuspension after drug sequestration. Further work is planned to investigate the effect ofliposomes in TCA toxicity in an animal model.
\end{abstract}

Keywords: Liposomes, antidepressants, tricyclic, toxicology, poisoning

\section{Background}

There has been much recent interest in the use of intravenous lipid based products in the treatment of tricyclic antidepressant (TCA) toxicity. New treatment modalities are of importance as TCA toxicity remains a major cause of mortality from poisoning [1]. Hypertonic sodium bicarbonate solution remains the mainstay of specific antidotal therapy. Intravenous lipid emulsions have been demonstrated to be effective in animal models, with clinical case reports suggesting additive benefit in TCA toxicity when sodium bicarbonate has not reversed shock $[2,3,4]$.

The initial experimental discovery of lipid emulsion as antidote was the result of structured observation rather than drug design. The proposed mechanism of action of lipid emulsions is pharmacokinetic - creating a new high affinity blood compartment into which the toxin redistributes. Recognition of potential for higher affinity lipid based toxin carriers has led to the development of toxin specific liposomes with higher affinity for TCA's than lipid emulsions. Those used in the present study are spherical phospholipid bilayers coated with polyethylene glycol to diminish interaction with plasma proteins. As weak bases, TCA's have been shown to bind to the surface of the liposomes due to electrostatic interaction with the charged phospholipids accompanied by hydrophobic interaction with the liposome annulus [5].
Liposomal formulations have been shown to be effective in sequestering TCA from plasma proteins in vitro $[6,7]$. At present there is no in vitro demonstration that liposomes bind TCA in the presence of red blood cells.

Liposomes are also of a size and uniformity that brings theoretical potential for removal from the circulation once toxin is bound. It is the purpose of the present study to pilot investigation into the binding of imipramine by liposomes in a suspension of human albumin and red blood cells and removal of these liposomes from solution once imipramine is bound. The filtering process by which liposomes and bound drug is removed from solution involves simulation of a clinically utilised plasmapharesis process.

\section{Method}

An ethical waiver was obtained from the Hunter New England Clinical Ethics Committee.

Constitution of blood/albumin/imipramine suspension Imipramine in solution (Sigma Aldrich) was added to a suspension comprised of $40 \% / 60 \%$ human red blood cells (expired for clinical use)/human albumin (4\% concentration, CSL Bioplasma). The target imipramine concentrations in the end suspension were 20 micromole/litre and 40 micromole/litre, these being concentrations above those seem in human case reports of fatal imipramine 

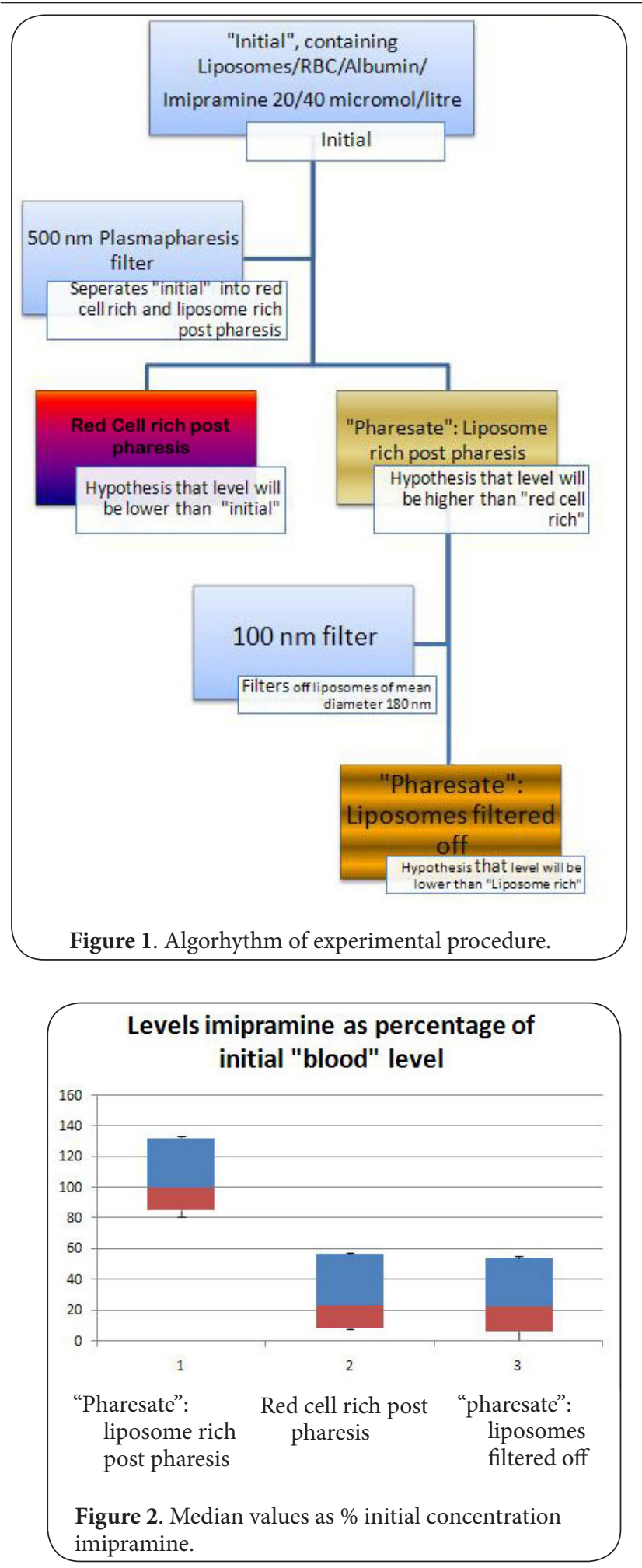

toxicity [8]. That these were the concentrations present in the initial study suspensions was confirmed by measuring levels at this stage of the experiment.

\section{Liposome preparation}

1,2-Dioleoyl-sn-glycero-3-[Phospho-rac-(1-glycerol)] and
1,2-Dipalmitoyl-sn-glycero-3-Phosphoethanolamine-N[Methoxy(Polyethylene glycol)-2000] (Sigma pharmaceuticals, USA) were combined using the method described previously for liposome manufacture [4] in University of Otago Department of Pharmacy. Mean liposome diameter was $180 \mathrm{~nm}$. Liposomes were added to $500 \mathrm{ml}$ of the blood/ albumin/toxin suspension at a concentration of 2 grams per litre.

\section{Plasmapharesis}

The blood/albumin/imipramine/liposome suspension was placed in a standard inflatable pressure bag and pressurised to $250 \mathrm{mmHg}$. This was attached via standard fashion to a plasmapharesis filter of pore size $500 \mathrm{~nm}$ (Infomed, Switzerland ). The purpose of this phase was to divide the suspension into red cell rich and liposome rich, red cell depleted components. The liposome rich component was then passed through a $100 \mathrm{~nm}$ filter (Merck millipore) intended to filter off liposomes along with any bound drug from this suspension. A schematic of the experimental setup is presented pictorially in Figure 1.

\section{Imipramine estimation}

Imipramine levels in each of the suspensions were measured after acetonitrile extraction of all drug bound to protein, red cells and liposomes (Chromatography unit, Royal Prince Alfred ospital, Sydney, Australia ). Acetonitrile extraction from liposomes was confirmed by measuring a known level of drug from a solution containing liposomes.

\section{Statistical analysis}

The primary outcome variable was percentage of initial imipramine concentration in the end suspensions. Previous work suggests a very high affinity of the liposomes for imipramine and large effect sizes were expected. Non Gaussian distributions were expected. Given these two factors, a minimum of four samples were taken across the two concentrations at each stage as this is the minimum number where statistical significance can be demonstrated using a rank sum test. The sole test used in the study was the Mann Whitney. Statistical analysis was undertaken using Graphpad Instat (r).

\section{Results}

Results are displayed graphically in Figure 2. The bars are a percentage of concentration of imipramine in pre plasmapharesis solution. One of the lab values for the "pharesate", liposomes filtered off group returned from the lab was 0 . While it was possible that this was either a lab or sample error this value was included in the analysis in keeping with an "intention to treat" principal.

Median values as \% initial concentration imipramine Figure 2 shows a box and whisker plot for suspensions at different points in the protocol. Significant differences were seen between the liposome rich post plasmapharesis (median 100\%, IQR 84-135\%) and both the red cell rich post 
Cave et al. Journal of Pharmaceutical Technology \& Drug Research 2013,

plasmapharesis (median, 24\%, IQR 9-43\%) $(p=0.002)$ and "pharesate", liposomes filtered off (median 23\%, IQR 9-37\%) $(p=0.03)$. These results are consistent with sequestration of imipramine by the liposomes with their subsequent filtration off in the cascaded process outlined in Figure 1.

\section{Discussion}

Our results suggest significant binding of imipramine by liposomes in the blood/albumin suspension. This is the first demonstration of such an effect in a solution containing human red blood cells. The effect of liposomes in overdose has been previously modelled using physiologically based pharmacokinetic modelling [6]. This work suggests that liposomes at a concentration of $2 \mathrm{gm} /$ litre could have significant clinical effects in TCA overdose. Our work confirms the plausibility of liposomes as drug binders in human blood.

Part of the filtration process for the liposomes involved a clinically used plasmapharesis technique. This raises the theoretical possibility of extracorporeal removal of liposomes once toxin is bound in the setting of a clinical overdose. While TCA toxicity was the second leading cause of death from medication intoxication in the decade 2002009 fatality was relatively rare with 80 deaths in 2007-2008 in the United States [1]. This means any extracorporeal removal technique would have to make use of already widely available hardware and techniques.

This study has a number of limitations. Albumin solution was used as a surrogate for plasma proteins due to availability. Additionally, the concentration of albumin at $2.4 \%$ was low relative to blood concentration. There is however, work using plasma protein solutions suggesting liposomes are effective at sequestering toxin $[\mathbf{5}, \mathbf{6}]$. Adverse effects of liposomes - particularly haemolysis - were not measured in this protocol, but there is a considerable literature regarding the biocompatibility of pegylated liposomes [6]. The model used for extracorporeal removal can demonstrate only theoretical feasibility. The flow and filtration rates through a standard plasmapharesis circuit are such that, even with a contracted volume of distribution resultant to liposome usage, the proportion of toxin removed relative to total body toxin load would be unlikely to be clinically relevant. Issues of flow rate through the extracorporeal circuit and removal fraction of liposomes per unit of blood through the circuit would require a remedy before consideration could be given to clinical use of this technique for extracorporeal removal of toxin. Liposomes composed of 95 molar percent of DOPG may not be ideal model to clinical use, as liposomes comprised of unsaturated phospholipids have shorter circulation times [9].

\section{Conclusions}

We have demonstrated significant experimental binding of imipramine to liposomes in a human albumin/red blood cell solution, and have removed bound imipramine from this solution after drug sequestration utilising a clinically available plasmapharesis technique. Further work is planned to investigate the effect of liposomes in TCA toxicity in an animal model.

\section{Competing interests}

The authors declare that they have no competing interests.

\section{Authors' contributions}

The study was initially conceived by Dr Cave.

Dr's Cave, Raghavan, Burrows and Harvey

designed study methodology.

Professor Chauhan designed the liposomal formulation and aided the manufacture process for liposomes.

Dr's Cave and Raghavan undertook the study protocol.

Dr's Cave and Harvey analysed study results.

Dr Cave prepared the initial draft of the manuscript.

All authors contributed to manuscript review.

\section{Funding discloser}

Liposome manufacture and Merck Millipore

filters were self funded by the lead author.

Plasmapharesis filter donated by Baxter, Australia.

Laboratory results funded by the University of

Newcastle Department of Rural Health.

\section{Acknowledgement}

Assoc Professor Sarah Hook, School of Pharmacy, University of Otago, assisted with liposome manufacture. Mr Tim Constable, equipment nurse, Tamworth Rural Referral Hospital, assisted with the study protocol.

\section{Publication history}

Received: 05-Dec-2012 Revised: 01-Jan-2013

Accepted: 10-Jan-2013 Published: 19-Feb-2013

\section{References}

1. Tsai V and Arabar A: Tricyclic Antidepressant Toxicity in Emergency Medicine. | Website

2. Harvey $M$ and Cave $G$ : Case report: successful lipid resuscitation in multi-drug overdose with predominant tricyclic antidepressant toxidrome. Int J Emerg Med 2012, 5:8. | Article | PubMed Abstract | PubMed Full Text

3. Blaber MS, Khan JN, Brebner JA and McColm R: "Lipid rescue" for tricyclic antidepressant cardiotoxicity. J Emerg Med 2012, 43:465-7. I Article | PubMed

4. Hendron D, Menagh G, Sandilands EA and Scullion D: Tricyclic antidepressant overdose in a toddler treated with intravenous lipid emulsion. Pediatrics 2011, 128:e1628-32. I Article I PubMed

5. Howell BA and Chauhan A: Interaction of cationic drugs with liposomes. Langmuir 2009, 25:12056-65. | Article | PubMed

6. Howell BA and Chauhan A: Binding of imipramine, dosulepin, and opipramol to liposomes for overdose treatment. J Pharm Sci 2009, 98:3718-29. | Article | PubMed

7. Howell BA and Chauhan A: A physiologically based pharmacokinetic (PBPK) model for predicting the efficacy of drug overdose treatment with liposomes in man. J Pharm Sci 2010, 99:3601-19. | Article | PubMed

8. Toxinz poisons information, Imipramine. | Website

9. Immordino ML, Dosio F and Cattel L: Stealth liposomes: review of the basic science, rationale, and clinical applications, existing and potential. Int J Nanomedicine 2006, 1:297-315. | PubMed Abstract | PubMed Full Text

\section{Citation:}

Cave G, Raghavan M, Burrows J, Harvey M and Chauhan A: Liposomal binding of imipramine in human red cell/albumin solution with simulated plasmapharesis. journal of Pharmaceutical Technology and Drug Research 2013, 2:8. http://dx.doi.org/10.7243/2050-120X-2-8 